\title{
Corrections
}

\section{Correction: Sincich and Horton, Independent Projection Streams from Macaque Striate Cortex to the Second Visual Area and Middle Temporal Area}

In the article "Independent Projection Streams from Macaque Striate Cortex to the Second Visual Area and Middle Temporal Area," by Lawrence C. Sincich and Jonathan C. Horton, which appeared on pages 5684-5692 of the July 2, 2003 issue, the authors discovered an error in the calculation of cell body size. On re-analysis, the cell body areas are larger by a factor of 10.45, for all populations. Thus, the median cell areas reported on p. 5687 should be $78 \mu \mathrm{m}^{2}$ for the cells projecting from V1 to V2, $203 \mu \mathrm{m}^{2}$ for V1 to MT projecting cells, and $161 \mu \mathrm{m}^{2}$ for the manifold V1 cells that project to V2 and MT. Values on the abscissa of Figure 5i should be multiplied by this scaling factor. None of the conclusions in the paper are altered.

DOI:10.1523/JNEUROSCI.4833-13.2013

\section{Correction: McKay et al., Sulcal Depth-Posiotion Profile Is a Genetically Mediated Neuroscientific Trait: Description and Characterization in the Central Sulcus}

In the article "Sulcal Depth-Posiotion Profile Is a Genetically Mediated Neuroscientific Trait: Description and Characterization in the Central Sulcus” by D. Reese McKay, Peter Kochunov, Matthew D. Cykowski, Jack W. Kent Jr, Angela R. Laird, Jack L. Lancaster, John Blangero, David C. Glahn, and Peter T. Fox, on pages 15618-15625 of the September 25, 2013 issue, the authors regret that Dr. Matthew D. Cykowski and Dr. Jack W. Kent Jr's affiliations were switched. Their corrected affiliation is as follows: Dr. Matthew D. Cykowski, ${ }^{6}$ Department of Pathology, University of Oklahoma Health Sciences Center, Oklahoma City, Oklahoma 73104, and Dr. Jack W. Kent Jr, ${ }^{5}$ Department of Genetics, Texas Biomedical Research Institute, San Antonio, Texas 78245.

DOI:10.1523/JNEUROSCI.4840-13.2013 